\title{
Evidence of Human Subthalamic Nucleus Involvement in Decision Making
}

\author{
Diane Whitmer ${ }^{1,2}$ and Corey N. White ${ }^{3}$ \\ ${ }^{1}$ NeuroTexas Institute at St. David's Healthcare, Austin, Texas 78705, ${ }^{2}$ Imaging Research Center and ${ }^{3}$ Department of Psychology, University of Texas, \\ Austin, Texas 78759 \\ Review of Zaghloul et al.
}

Deciding between multiple appealing (or unappealing) options is sometimes difficult, and the neural mechanisms of this process are not yet fully understood. Recently, experimental studies and computational models have explored the potential roles of the basal ganglia in mediating decision making through reciprocal connections with cortex. Some of these studies specifically propose that the subthalamic nucleus (STN) "holds off" cortically mediated action selection, allowing integration of more information for making difficult decisions (Frank, 2006; Frank et al., 2007).

Because intraoperative electrophysiology is typically used to identify the STN as a target structure for deep brain stimulation (DBS) implantation for Parkinson's disease, it is possible to measure the neural activity from the STN while the patient is awake and able to engage in tasks. In a recent study published in The Journal of Neuroscience, Zaghloul and colleagues (2012) used this approach to test the hypothesis that the neural activity of the STN encodes the degree of "decision conflict," that is, the difficulty of choice between options with similar reward values. The authors recorded single-unit activity from the STN of patients undergoing DBS surgery

\footnotetext{
Received April 14, 2012; revised May 16, 2012; accepted May 21, 2012. We thank Dr. Teresa Esch for her helpful editorial comments. Correspondence should be addressed to Dr. Diane Whitmer, NeuroTexas Institute at St. David's Healthcare, 1015 E. 41st Street, Suite 404, Austin, Texas 78705. E-mail: dwhitmer@austin.utexas.edu.

DOI:10.1523/JNEUROSCI.1819-12.2012

Copyright $\odot 2012$ the authors $\quad 0270-6474 / 12 / 328753-03 \$ 15.00 / 0$
}

while the patients performed a rewardconflict decision-making task. During the training phase of the task, subjects were presented with a series of trials in which they chose one of two simultaneously presented visual symbols via a right- or left-hand button press. Three pairs of symbols were presented during training, and for a given symbol in the pair, the probability of receiving a reward was fixed and probabilistic (ranging from 20\% to $80 \%$ ). After each choice, visual and auditory feedback indicated whether the choice was "correct" (rewarded) or "incorrect." Based on this feedback, participants learned over time which symbols were more likely to result in reward. The learned "reward expectation" for each symbol was defined as the rate of selection of a particular symbol during the final block of training; i.e., symbols that were expected to lead to reward were selected more often.

During the testing phase of the task, trials consisted of the originally trained symbol pairs in addition to new pairs constructed by rearranging the symbols into new pairings. For example, if $\mathrm{A}-\mathrm{B}$ and $\mathrm{C}-\mathrm{D}$ were trained, test pairs would include $\mathrm{A}-\mathrm{B}, \mathrm{C}-\mathrm{D}, \mathrm{A}-\mathrm{C}$, and $\mathrm{B}-\mathrm{D}$. These new pairings resulted in a wide range of reward expectations across the different symbol pairs. In the testing phase, no feedback was presented after the choice. Decision conflict was defined as one minus the absolute value of the difference in reward expectations between two stimuli. Thus, if both symbols had a similarly high (or low) expectation of reward, there would be high decision conflict and it would be more difficult to decide between the two options. This measure of decision conflict was used to probe STN activity.

The authors found that STN spiking increased in the 100-400 ms window after visual stimuli were presented, and this increase was greater for high-conflict stimulus pairs than for low-conflict pairs. The increased firing rate during this time window also correlated with response time, whereby smaller differences in expected reward (i.e., high conflict) corresponded to behavioral slowing. This is not surprising given that increased response time is a typical behavioral correlate of increased difficulty.

Close examination of Figure $3 d$ of Zaghloul et al. (2012) suggests that of the 15 possible reward expectation pairs, six of which involved high $(>0.8)$ decision conflict, only two in particular were responsible for the statistical effect of increased spike rate as a function of increased decision conflict. Because the association between fixed reward probabilities and specific stimuli were randomized across patients, this result cannot be explained by outliers within the set of visual stimuli. It would be interesting to know which reward value pairs were responsible for this positive correlation. For example, was the correlation between increased spiking and decision conflict explained by pairs with similarly high reward expectation (e.g., win/win trials with $80 \%$ vs $85 \%$ reward) or by pairs with similarly low reward expectation (e.g., lose/lose trials with 
$20 \%$ vs $25 \%$ reward)? This is an important distinction because previous research has demonstrated that Parkinson's patients' abilities to learn correct choices more easily from positive versus negative reinforcement are based on their levels of dopamine (Frank et al., 2004). Although Zaghloul and colleagues (2012) found no statistical difference in spike rates between win/win, win/ lose, and lose/lose trial types, the correlation between spike rate and decision conflict could have a dependency on expectation valence.

\section{What does STN spiking encode?}

The task used by Zaghloul et al. (2012) is a complex one, likely engaging multiple cognitive processes, including visual recognition, memory retrieval, action selection, reward anticipation, movement preparation, and motor action. The design of the study, despite controlling for many variables, cannot disambiguate all of these factors. Behavioral results demonstrated that patients successfully learned the reward values of only a subset of the stimuli. If these were the stimuli pairs responsible for the statistically increased spiking displayed in Figure $3 d$, then it is possible that the increased spiking encoded successful memory retrieval or learning rather than decision conflict. A comparison of spiking responses from the final training phase and from the testing phase might in theory aid the dissociation of some of these factors because memory retrieval and learning would play a lesser role during the initial training session, but the data for this comparison were not available.

A separate study with a similar behavioral paradigm was performed recently with local field potential (LFP) recordings from the DBS lead in the STN (Cavanagh et al., 2011). Compared with low-conflict trials, high-conflict trials exhibited lower theta power within $250 \mathrm{~ms}$ and higher delta band power $750 \mathrm{~ms}$ after stimulus presentation. The relationship between the spiking observed by Zaghloul et al. (2012) and this modulation of low-frequency power is unclear. A correspondence between spiking and $13-35 \mathrm{~Hz}$ beta band power was previously demonstrated by concurrently measured LFP and spike recordings in the STN, particularly in the dorsal region of the STN and during tremor (Kühn et al., 2005). Zaghloul et al. (2012) recorded spiking from the ventral region of the STN and likely controlled for tremor signals by normalizing to baseline spiking rates. In some cases, the theta rhythm can serve as an envelope that modulates spiking (Harris et al., 2002). The increased spiking $200 \mathrm{~ms}$ after the stimuli in high-conflict trials reported by Zaghloul et al. (2012) might therefore represent the alignment of local spiking to a theta trough generated by population level activity and cortico-STN dynamics.

\section{What is the evidence for the STN as a modulator of decision thresholds?}

Zaghloul et al. (2012) suggest that the STN is well suited to "adjust threshold criteria" for cortical firing rate models of decision making. This predicts a causal link between STN and behavioral slowing resulting from increased response caution, but support for this hypothesis is not evident in the present study. Because multiple areas of cortex connect monosynaptically to the STN, cortical areas could just as easily adjust threshold criteria for STN-mediated decisions as vice versa. Even if the STN's increased spiking does convey a message to cortex in response to decision difficulty, there are a number of possible messages that this could be. For example, increased spiking could signify a "hold your horses" signal to allow more time for evidence accumulation, resulting in behavioral slowing beyond what occurs with increased difficulty (Frank, 2006). Alternatively, it might signal "bring more attentional resources" to downstream nuclei such as the thalamus.

Previous behavioral results from Parkinson's patients off medication suggested that these patients have greater difficulty deciding between two negative (lose/lose) choices than deciding between two positive (win/ win) choices, increasing their decision threshold to slow their responses and allow greater evidence accumulation (Frank et al., 2004). If this relationship holds in the study by Zaghloul and colleagues (2012), there should be two sources of behavioral slowing: slowing resulting from difficulty, and additional slowing for trials with two negative choices (resulting from the "hold your horses signal"). This prediction was not borne out. The authors found that there was no correlation between response time and reward pair type. Moreover, spike rates were no greater for decision conflict from two unappealing choices than from two appealing choices. The discrepancy between these results and the behavioral slowing model might be explained by several factors. Because the patients stopped medication only $12 \mathrm{~h}$ before surgery, they may not have been fully in the "off" state. Some Parkinson's medications are long-acting and require several days to be fully removed from the patient's system. Alternatively, the lack of increased spiking during trials with two negative choices might simply reflect that the patient was guessing because of the low learning rates for many of the stimuli. Importantly, the only behavioral slowing found in the present study can be completely attributed to a natural consequence of increased difficulty (i.e., slower evidence accumulation); thus, there is little evidence for a link between these STN recordings and threshold adjustment as described by Frank and colleagues (Frank et al., 2004; Frank, 2006).

\section{Future directions}

Invasive intraoperative recordings from deep structures of the human brain involve inherent experimental challenges such as an electrically noisy environment, severe experimental time restrictions, and numerous uncontrollable factors pertaining to the patient's behavioral state. That the study demonstrated task-specific changes to STN firing rates based on limited data is interesting. The question remains as to whether an increase in STN firing rate is causal or incidental to decision conflict, and whether these firing rates are functionally related to changes in decision thresholds. Although ethical considerations limit the options for interventions, the use of dopaminergic medication or DBS to exogenously alter the firing rate might provide opportunities to test the causality of STN firing patterns during decision conflict. Although the recording of single units during DBS would be difficult because of saturation of the amplifiers by the stimulation artifact, the recording of local field potentials during DBS is feasible (Rossi et al., 2007). This approach could potentially provide the opportunity to safely perturb the system and measure the simultaneous effects on neural and behavioral responses, further elucidating the role of STN activity in decision conflict.

\section{References}

Cavanagh JF, Wiecki TV, Cohen MX, Figueroa CM, Samanta J, Sherman SJ, Frank MJ (2011) Subthalamic nucleus stimulation reverses mediofrontal influence over decision threshold. Nat Neurosci 14:1462-1467.

Frank MJ (2006) Hold your horses: a dynamic computational role for the subthalamic nucleus in decision making. Neural Netw 19:1120-1136.

Frank MJ, Seeberger LC, O’reilly RC (2004) By carrot or by stick: cognitive reinforcement learning in parkinsonism. Science 306:1940-1943

Frank MJ, Samanta J, Moustafa AA, Sherman SJ (2007) Hold your horses: impulsivity, deep brain stimulation, and medication in parkinsonism. Science 318:1309-1312. 
Harris KD, Henze DA, Hirase H, Leinekugel X, Dragoi G, Czurkó A, Buzsáki G (2002) Spike train dynamics predicts theta-related phase precession in hippocampal pyramidal cells. Nature 417:738-741.

Kühn AA, Trottenberg T, Kivi A, Kupsch A, Schneider GH, Brown P (2005) The relationship between local field potential and neuronal discharge in the subthalamic nucleus of patients with Parkinson's disease. Exp Neurol 194:212-220.

Rossi L, Foffani G, Marceglia S, Bracchi F, Barbieri S, Priori A (2007) An electronic device for artefact suppression in human local field po- tential recordings during deep brain stimulation. J Neural Eng 4:96-106.

Zaghloul KA, Weidemann CT, Lega BC, Jaggi JL, Baltuch GH, Kahana MJ (2012) Neuronal activity in the subthalamic nucleus encodes decision conflict during action selection. J Neurosci 32:2453-2460. 